\title{
Cigarette smoke and bleomycin-induced pulmonary oxidative stress in rats
}

\author{
TURGUT TEKE ${ }^{1}$, EMIN MADEN ${ }^{1}$, AYSEL KIYICI ${ }^{2}$, CELALETTIN KORKMAZ $^{1}$, \\ MEHMET GOK $^{1}$, FARUK OZER ${ }^{1}$, OKTAY IMECIK ${ }^{1}$ and KURSAT UZUN ${ }^{1}$ \\ Departments of ${ }^{1}$ Chest Diseases and ${ }^{2}$ Biochemistry, Meram Medical Faculty, \\ Konya University, 42080 Meram-Konya, Turkey
}

Received February 26, 2012; Accepted April 5, 2012

DOI: $10.3892 /$ etm. 2012.550

\begin{abstract}
Bleomycin causes pulmonary fibrosis by increasing free oxygen radicals. Cigarette smoke is a strong oxidant which adversely affects pulmonary tissue. We evaluated the effects of cigarette smoke administered with intratracheal bleomycin on pulmonary tissue. We studied 3 groups of rats $(n=10)$ : one group received intratracheal saline and served as a control; one received intratracheal bleomycin (IT) $(0.5 \mathrm{U} / 100 \mathrm{~g}$ body weight, single dose on the first day), and one group received intratracheal bleomycin (single dose on first day) and tobacco smoke (two times per day) (IT-S). After 4 weeks, the levels of malondialdehyde (MDA) and nitric oxide (NO) and the activities of superoxide dismutase (SOD) and xanthine oxidase (XO) were assayed in the homogenate of the lung tissue samples. The severity of interstitial fibrosis was assessed using the grading system described by Ashcroft. There was more intensive fibrosis in the IT and IT-S than in the control samples $(\mathrm{P}<0.001)$. The levels of MDA, NO and activity of XO were significantly increased $(\mathrm{P}<0.001,<0.002$ and $<0.002$, respectively), and SOD activity $(\mathrm{P}<0.001)$ was significantly decreased in the IT group when compared to these values in the control group. The concentration of NO was significantly decreased $(\mathrm{P}<0.002)$, and SOD activity was significantly increased $(\mathrm{P}<0.05)$ in the lung tissue samples of the IT-S group. Theoretically, the combination of cigarette smoke and bleomycin may have a synergistic effect on oxidative lung injury. In conclusion, we showed that inhalation of cigarette smoke provides protection against oxidative stress in the lung tissue of rats with bleomycin-induced pulmonary fibrosis.
\end{abstract}

\section{Introduction}

Interstitial pulmonary fibrosis is thought to arise as a result of a response to persistent lung injury and inflammation.

Correspondence to: Dr Turgut Teke, Department of Chest Diseases, Meram Medical Faculty, Konya University, Gogus Hastaliklari Anabilim Dali, 42080 Meram-Konya, Turkey

E-mail: turgutteke@hotmail.com

Key words: bleomycin, oxidative stress, pulmonary fibrosis, cigarette smoke
An oxidant-antioxidant imbalance in the lower respiratory tract has been proposed as a cause of pulmonary fibrosis (1). Cigarette smoking is causally related to the development of desquamative interstitial pneumonia, pulmonary Langerhans cell histiocytosis and idiopathic pulmonary fibrosis (IPF). However, cigarette smoke exposure (CSE) appears to protect against the development of hypersensitivity pneumonitis, a lymphocytic alveolitis provoked by the exposure to organic particles, and sarcoidosis, an inflammatory disorder of unknown etiology (2). The mechanisms by which CSE affects in one or another manner the inflammatory and fibrotic responses in the lung remain to be elucidated. Smoking is a strong oxidant which has a role in the development of pulmonary fibrosis (3).

Bleomycin (BLM) causes pulmonary fibrosis by increasing the free oxygen radical content which causes severe pulmonary damage. An imbalance between oxidants and antioxidants has been proposed in the pathogenesis of BLM-induced lung fibrosis. BLM-induced free oxygen radical production has been shown to stimulate fibroblasts to secrete collagen, and to proliferate and differentiate into myofibroblasts, resulting in a histological appearance that is similar to IPF (4).

The purpose of this study was to evaluate the effect of smoking on oxidative injury in lung tissue of rats with BLM-induced pulmonary fibrosis.

\section{Materials and methods}

Adult male Sprague-Dawley rats (body weight, 315-375 g) were maintained at $22^{\circ} \mathrm{C}$ and $20-50 \%$ humidity, with a 12 -h light period and supplied with a standard laboratory chow diet and water ad libitum. The animals were assigned to three groups of rats $(n=10)$. One group, the control, received intratracheal normal saline; another group (IT) received intratracheally one dose of BLM (Nippon Kayaku Co., Ltd., Tokyo, Japan) $0.5 \mathrm{U} / 100 \mathrm{~g}$ body weight in $0.3 \mathrm{ml} 0.15 \mathrm{M}$ sterile $\mathrm{NaCl}$. The rats in the third group received intratracheal BLM and were later placed in a plastic cage $(40 \mathrm{~cm} \times 26 \mathrm{~cm} \times 16 \mathrm{~cm})$ two times a day and exposed to tobacco smoke for 4 weeks. BLM solution was prepared immediately before administration and was administered intraperitoneally directly into the trachea under light anesthesia with sodium pentobarbital $(50 \mathrm{mg} / \mathrm{kg}$ body weight). 
After 4 weeks, the animals were sacrificed at the end of the experiment. The right lungs were removed and were shock frozen immediately after resection and were stored at $-80^{\circ} \mathrm{C}$ until use. Each lung was excised, rinsed in ice-cold physiological saline and homogenized in Tris-HCl buffer, using a tissue homogenizer. The resultant whole tissue homogenate was used for biochemical measurements.

The levels of malondialdehyde (MDA), nitric oxide (NO) and the activity superoxide dismutase (SOD), xanthine oxidase $(\mathrm{XO})$ in the tissue samples were analyzed using the following methods.

The MDA level was estimated by the double-heating method of Wasowicz et al (5). The concentration of MDA was calculated by the absorbance coefficient of the MDA-TBA complex (absorbance coefficient e $=1.56 \times 10^{5} \mathrm{l} / \mathrm{mol} / \mathrm{cm}$ ) and expressed as $\mathrm{nmol} / \mathrm{g}$ for the lung. Total SOD activity $(\mathrm{Cu} / \mathrm{Zn}$ and $\mathrm{Mn}$ ) was determined using the method of Sun et al (6). Activity was expressed as U/mg for the lung.

NO was measured using a colorimetric kit method (cat. no. CM780001, Cayman Chemical Co., USA). The concentration of $\mathrm{NO}$ was expressed as $\mu \mathrm{M} / \mathrm{g}$ protein. XO activity was determined by the method of Prajda and Weber (7). This activity was expressed as $\mathrm{U} / \mathrm{g}$ protein.

After sacrifice, each left lung was fixed in a buffered $10 \%$ formalin solution for $24 \mathrm{~h}$ and embedded in paraffin. Longitudinal sections of the lungs were stained with hematoxylin and eosin (H\&E) and were examined for pulmonary fibrosis. Each successive field was individually assessed for the severity of interstitial fibrosis using the semi-quantitative grading system described by Ashcroft et al (8). The entire lung section was reviewed at a magnification of x100. For each of the 30-35 microscopic fields needed to review the section, a score ranging from 0 (normal) to 8 (total fibrosis) was assigned.

Statistical analysis. Results are expressed as the means \pm SD. A non-parametric analysis of variance (Kruskal-Wallis method) with post-hoc Bonferroni's correction was used to determine any significant variance among the five groups. The Mann-Whitney U test was performed for comparison between groups. All analyses were carried out using the SPSS statistical software package, and a probability value of $<0.05$ was considered to be statistically significant.

Ethical approval. Ethical approval was provided from the Ethics Committee of Meram Medical Faculty, affiliated at the time of the study to Selcuk University, Konya, Turkey.

\section{Results}

There was more intensive fibrosis in the IT and IT-S groups than that in the control samples $(\mathrm{P}<0.001)$. There was no significant difference between the IT and IT-S groups with respect to fibrosis score $(\mathrm{P}>0.05)$.

The results of oxidative stress marker levels of the control, IT and IT-S groups are shown in Table I and Fig. 1. When compared with the control group, oxidative stress was increased significantly in the BLM-administered IT group $(\mathrm{P}<0.05)$. When the effect of smoking on oxidative stress in the BLM-treated groups was evaluated, a significant increase in SOD activity and a significant decrease in NO level (deter-
Table I. Levels of oxidative stress markers in lung tissue.

\begin{tabular}{lrrrr}
\hline \multicolumn{5}{c}{ Lung } \\
\cline { 2 - 5 } Group & \multicolumn{1}{c}{ SOD } & MDA & NO & XO \\
\hline C & $0.3 \pm 0.07$ & $4.5 \pm 0.7$ & $0.18 \pm 0.05$ & $1.39 \pm 0.5$ \\
IT & $0.17 \pm 0.04$ & $9.9 \pm 2.8$ & $0.57 \pm 0.2$ & $2.7 \pm 0.7$ \\
IT-S & $0.22 \pm 0.04$ & $8.9 \pm 2.9$ & $0.21 \pm 0.1$ & $2.2 \pm 0.7$
\end{tabular}

$\mathrm{C}$, control group; IT, intratracheal bleomycin; IT-S, intratracheal bleomycin + tobacco smoke.

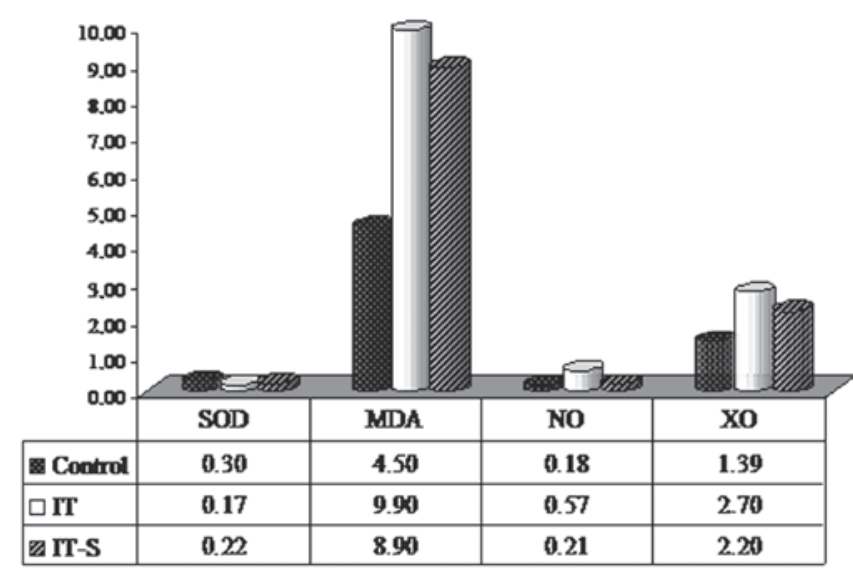

Figure 1. SOD and XO activity and MDA and NO levels in lung tissue of the control, intratracheal bleomycin (IT) and intratracheal bleomycin + tobacco smoke (IT-S) groups.

Table II. P-values for comparison between groups.

\begin{tabular}{lcccc}
\hline & SOD & MDA & NO & XO \\
\hline C vs. IT & $<0.001$ & $<0.001$ & $<0.002$ & $<0.002$ \\
IT vs. IT-S & $<0.05$ & NS & $<0.002$ & NS \\
\hline C, control group; IT, intratracheal bleomycin; & IT-S, intratracheal bleo- \\
mycin + tobacco smoke; NS, no significant.
\end{tabular}

minants of decreased oxidative stress) in the IT-S group compared to the IT group. There was no statistically significant decrease in MDA level and XO activity in the IT-S group when compared to the IT group. In Table II a comparison between groups carried out with the Mann-Whitney U test is shown.

\section{Discussion}

The results of the present study indicated increased oxidative stress in the lung tissue of rats that developed after BLM-induced pulmonary fibrosis, and CSE reduced the oxidative injury induced by BLM.

There is limited information on the role of tobacco smoking in the development and outcome of BLM-induced 
lung fibrosis. The mechanisms by which CSE affects in one way or another the incidence or severity of various interstitial lung diseases are not entirely clear (3). Several studies have aimed to reveal whether a smoking history increases the risk of BLM-induced lung fibrosis. An increased risk in smokers is strongly suggested by Lower et al (9). These authors showed radiographic alterations consistent with BLM-induced lung fibrosis in 55\% of smoking patients receiving BLM compared with $0 \%$ of non-smokers. An earlier report by Takada et al (10) found that an intratracheal administration of BLM induced fibrotic changes in the lungs of hamsters exposed to cigarette smoke (CS). It was also shown that exposure to CS increased the number of myofibroblasts in alveolar septa of guinea pigs and potentialized pulmonary injury induced by BLM (3).

Various studies have shown a high percentage of ever smokers among individuals with IPF and that CSE is an independent risk factor for the development of $\operatorname{IPF}(11,12)$. However, cigarette smoking has only recently been shown to be associated with improved survival in this disease (13). Osanai et al (14) found that tobacco smoke reduced the fibrotic response to BLM. In addition, a number of clinical and experimental studies suggest that CSE reduces the frequency of radiation-induced pneumonitis $(15,16)$.

Theoretically, the combination of CS inhalation and BLM should have a synergistic effect on the development of BLM-induced lung injury. We hypothesized that CS contributes to the development and outcome of BLM-induced lung fibrosis by increasing oxidative stress. Our study tested this hypothesis in rats, and we observed that in contrast to this hypothesis, CSE had no additional effect on the development of fibrosis.

Reduced oxidative stress after CS exposure in rats with BLM-induced lung injury can be explained by hyperoxia and reduced oxidative injury caused by hyperoxia. Firstly, one of the few indicators of improved prognosis in IPF is active cigarette smoking at the time of diagnosis. This phenomenon may be related to the $\sim 11 \mathrm{mg}$ of carbon monoxide (CO) (or $1-6 \%$ CO gas) inhaled with each cigarette (17). Secondly, smokers are now known to have increased airway expression of heme oxygenase-1 (HO-1). HO-1 catalyzes heme degradation to generate $\mathrm{CO}$, biliverdin and free iron. Extensive data suggest that CO can often substitute for HO-1 (18). The toxic properties of $\mathrm{CO}$ are well known in the field of pulmonary medicine. The toxic actions of $\mathrm{CO}$ relate to its high affinity for hemoglobin (240-fold greater than that of $\mathrm{O}_{2}$ ). CO replaces $\mathrm{O}_{2}$ rapidly from hemoglobin, causing tissue hypoxia (19). Only recently has it become known that, at very low concentrations, $\mathrm{CO}$ participates in many physiological reactions. $\mathrm{CO}$ exposure of 100-250 parts per million (ppm) stimulate physiological effects without apparent toxicity. The majority of endogenous $\mathrm{CO}$ production originates from active heme metabolism. CSE represents a major source of chronic low level exposure to $\mathrm{CO}$ (20).

Inhaled CO initially targets alveolar macrophages and respiratory epithelial cells. $\mathrm{CO}$ has been shown to decrease proliferation of fibroblasts (21). CO may limit the generation of ROS, lower the presence of free metal ions and downregulate pro-inflammatory cytokines. Exogenous administration of low concentrations of $\mathrm{CO}$ provided protection against oxidative stress in a model of inflammation (22). A low concentration of
$\mathrm{CO}$ was also found to provide protection from lipopolysaccharide-induced injury via directly inhibiting lipid peroxidation and decreasing ROS. Liu et al (23) investigated the effect of $\mathrm{CO}$ inhalation on oxidative stress and demonstrated that the MDA level and myeloperoxidase activity increased significantly in rats with lung injury related to lipopolysaccharide; and $\mathrm{CO}$ inhalation significantly decreased the MDA and myeloperoxidase accumulation and significantly increased the SOD activity in lungs which indicates that $\mathrm{CO}$ repairs oxidative lung injury.

Hyperoxia generates ROS, e.g., superoxide anion and hydrogen peroxide, which can injure the lung (24). Rats exposed to hyperoxia in the presence of a low concentration of $\mathrm{CO}$ exhibit less lung injury than control rats exposed to oxygen alone (25). A similar study by Clayton et al (26) demonstrated a statistically significant reduction in pulmonary edema upon exposure to $\mathrm{CO}$ and hyperoxia. Since BLM is assumed to induce its toxicity partially by the induction of free radicals, the administration of high inspired oxygen could be hazardous (27). In hamsters treated with BLM and 70\% oxygen for $72 \mathrm{~h}$, the mortality was $90 \%$ compared with $15 \%$ in those animals that received BLM only (28). Zhou et al (21) documented that $\mathrm{CO}$ may protect against BLM-induced lung injury in mice. Mice treated with $\mathrm{CO}$ and BLM were found to have less severe lung injury than mice treated with BLM alone. In humans, clear data showing an increased risk of BLM-induced lung injury with concomitant oxygen supplementation are lacking; however, because of the data obtained from animal studies, hyperoxia is discouraged during BLM treatment (29).

In conclusion, the inhalation of cigarette smoke, which is similar to the exogenous administration of low concentrations of $\mathrm{CO}$, can provide protection against oxidative stress in the lung tissue of rats with bleomycin-induced pulmonary fibrosis.

\section{References}

1. Kinnula VL, Fattman CL, Tan RJ and Oury TD: Oxidative stress in pulmonary fibrosis: a possible role for redox modulatory therapy. Am J Respir Crit Care Med 172: 417-422, 2005.

2. Murin S, Smith BK and Matthay R: Other smoking affected pulmonary diseases. Clin Chest Med 21: 121-137, 2000.

3. Cisneros-Lira J, Gaxiola M, Ramos C, Selman M and Pardo A: Cigarette smoke exposure potentiates bleomycin-induced lung fibrosis in guinea pigs. Am J Physiol Lung Cell Mol Physiol 285: L949-L956, 2003.

4. Vlalov SL, Gabbiani G and Kapanci Y: Rat alveolar myofibroblasts acquire alpha-smooth muscle actin expression during bleomycin-induced pulmonary fibrosis. Am J Pathol 143: 1754-1765, 1993.

5. Wasowicz W, Nève S and Peretz A: Optimized steps in fluorometric determination of thiobarbituric acid-reactive substances in serum: importance of extraction $\mathrm{pH}$ and influence of sample preservation and storage. Clin Chem 39: 2522-2526, 1993.

6. Sun Y, Oberley LW and Ying L: A simple method for clinical assay of superoxide dismutase. Clin Chem 34: 497-500, 1988.

7. Prajda $\mathrm{N}$ and Weber G: Malignant transformation-linked imbalace: decreased XO activity in hepatomas. FEBS Lett 59: 245-249, 1975.

8. Ashcroft T, Simpson JM and Timbrell V: Simple method of estimating severity of pulmonary fibrosis on a numerical scale. J Clin Pathol 41: 467-470, 1988.

9. Lower EE, Strohofer S and Baughman RP: Bleomycin causes alveolar macrophages from cigarette smokers to release hydrogen peroxide. Am J Med Sci 295: 193-197, 1988.

10. Takada K, Takahashki K, Sato S and Yasui S: Cigarette smoke modifies bleomycin-induced lung injury to produce lung emphysema. Tohoku J Exp Med 153: 137-144, 1987. 
11. Schwartz DA, Helmers RA, Galvin JR, et al: Determinants of survival in idiopathic pulmonary fibrosis. Am J Respir Crit Care Med 149: 450-454, 1994

12. Baumgartner KB, Samet J, Stidley CA, et al: Cigarette smoking: a risk factor for idiopathic pulmonary fibrosis. Am J Respir Crit Care Med 155: 242-248, 1997.

13. King TE, Tooze JA, Schwarz MI, Brown KR and Cherniack RM: Predicting survival in idiopathic pulmonary fibrosis: scoring system and survival model. Am J Respir Crit Care Med 164: $1171-1181,2001$

14. Osanai K, Takahashi K, Suwabe A, et al: The effect of cigarette smoke on bleomycin-induced pulmonary fibrosis in hamsters. Am Rev Respir Dis 138: 1276-1281, 1988.

15. Johansson S, Bjermer L, Franzen L and Henriksson R: Effects of ongoing smoking on the development of radiation-induced pneumonitis in breast cancer and oesophagus cancer patients. Radiother Oncol 49: 41-47, 1998.

16. Nilsson K, Henriksson R, Cai YQ, Hellstrom S, Hornqvist BS and Bjermer L: Effects of tobacco-smoke on radiation-induced pneumonitis in rats. Int J Radiat Biol 62: 719-727, 1992.

17. Jarvis MJ: Trends in sales weighted tar, nicotine, and carbon monoxide yields of UK cigarettes. Thorax 56: 960-963, 2001

18. Morse D: The role of heme oxygenase-1 in pulmonary fibrosis. Am J Respir Cell Mol Biol 29: S82-S86, 2003.

19. Piantadosi CA: Carbon monoxide poisoning. N Engl J Med 347: 1054-1055, 2002.
20. Vremen HJ, Wong RJ and Stevenson DK: Carbon monoxide in breath, blood, and other tissues. In: Carbon Monoxide Toxicity. Penney DG (ed). CRC Press, FL, pp19-60, 2000

21. Zhou Z, Song R, Fattman CL, et al: Carbon monoxide suppresses bleomycin-induced lung fibrosis. Am J Pathol 166: 27-37, 2005.

22. Ng CS, Wan S and Yim AP: Pulmonary ischemia-reperfusion injury: role of apoptosis. J Eur Res 25: 356-363, 2005.

23. Liu SH, Ma K, Xu B and Xu XR: Carbon monoxide inhalation protects lung from lipopolysaccharide-induced injury in rat. Sheng Li Xue Bao 58: 483-489, 2006.

24. Brigham KL and Meyrick B: Endotoxin and lung injury. Am Rev Respir Dis 133: 913-927, 1986.

25. Otterbein LE, Mantell LL and Choi AM: Carbon monoxide provides protection against hyperoxic lung injury. Am J Physiol 276: L688-L694, 1999.

26. Clayton CE, Carraway MS, Suliman HB, et al: Inhaled carbon monoxide and hyperoxic lung injury in rats. Am J Physiol Lung Cell Mol Physiol 281: L949-L957, 2001.

27. Blom-Muilwijk MC, Vriesendorp R, Veninga TS, et al: Pulmonary toxicity after treatment with bleomycin alone or in combination with hyperoxia. Br J Anaesth 60: 91-97, 1988.

28. Tryka AF, Skornik WA, Godleski JJ and Brain JD: Potentiation of bleomycin-induced lung injury by exposure to $70 \%$ oxygen. Am Rev Respir Dis 126: 1074-1076, 1982

29. Sleijfer S: Bleomycin-induced pneumonitis. Chest 120: 617-624, 2001. 